\title{
Effect of Temperature on the Critical Micelle Concentration and Micellization Thermodynamic of Nonionic Surfactants: Polyoxyethylene Sorbitan Fatty Acid Esters
}

\author{
EHSAN MOHAJERI ${ }^{1}$ AND GHOLAMREZA DEHGHAN NOUDEH ${ }^{* 2}$ \\ ${ }^{1}$ Faculty of Pharmacy \\ Kerman University of Medical Sciences, Kerman, Iran \\ ${ }^{2}$ Pharmaceutics Research Center, Kerman University of Medical Sciences, \\ Kerman, Iran \\ ghr_dehghan@kmu.ac.ir
}

Received 27 January 2011; Accepted 12 April 2011

\begin{abstract}
In this study, non-ionic surfactants, polyoxyethylene sorbitan fatty acid esters (polysorbate) are chosen to examine the temperature effect on the CMC over a wide temperature range. The enthalpy and entropy of micelle formation are evaluated according to the phase separation model. The surface tension of solutions was determined by means of Du Nöuys ring. The CMC values were taken from the sharp breaks in the surface tension vs. logarithms of surfactant concentration plots. As the surfactants' chain length increases the $\mathrm{CMC}$ at a constant temperature decreases, which is directly related to the decrease of hydrophilicity of the molecules. For each surfactant, as the system temperature increases, the CMC initially decreases and then increases, owing to the smaller probability of hydrogen bond formation at higher temperatures. The onset of micellization tends to occur at higher concentrations as the temperature increases. To evaluate the enthalpy of micellization, the CMCs are first correlated by a polynomial equation. It is found that $\Delta G^{\circ}{ }_{m}$ decreases monotonically as the temperature increases over the whole temperature range. Both $\Delta H^{\circ}{ }_{m}$ and $\Delta S^{\circ}{ }_{m}$ appear to be decrease monotonically with an increase in temperature. The compensation temperature was found to be $42{ }^{\circ} \mathrm{C}$ by linear regression over the whole temperature range and for all three surfactant systems together.
\end{abstract}

Keywords: Nonionic surfactant, Micellization thermodynamic, CMC.

\section{Introduction}

The capacity of aggregation in a solution is one of the characteristics of surfactants. As the aggregation formed, within a narrow concentration range, some physical properties of the surfactant solutions change abruptly. Micelles are one type of aggregation, and the narrow concentration range is called the critical micelle concentration (CMC), above which micelles are formed in the solutions. Several parameters of micellization such as aggregation number 
(n), and CMC can vary by changing the environmental conditions. Micellization is affected by various factors including surfactant nature (chain length, hydrophobic volume, and head group area), temperature, solvent, additive, pressure, $\mathrm{pH}$, ionic strength, etc ${ }^{1}$.

For ionic and amphoteric surfactants, micellization is affected by temperature as the hydrophobic and head group interactions change with temperature. Accordingly, CMC versus temperature studies have been performed to obtain information on these interactions ${ }^{2}$. For ionic surfactants in an aqueous solution, the CMC first monotonically decreases to a certain minimum around $25{ }^{\circ} \mathrm{C}$ while temperature is increasing temperature and then increases with further increase in temperature displaying a U-shaped behavior ${ }^{3}$.

In nonionic surfactants, CMC decreases as the temperature is increased. This is due to an increasing in the destruction of hydrogen bonds between water molecules and surfactanys hydrophilic groups. The log CMC versus $1 / T$ plot is nearly linear ${ }^{4}$. However, other studies are showing something else, for the nonionic surfactants such as polyoxyethylenated glycol monoether in an aqueous solution exhibited a minimum in the $\mathrm{CMC}$-temperature curve. The temperature of the minimum in the CMC-temperature curve is around $50{ }^{\circ} \mathrm{C}$ and increases as the oxyethylene chain length increases. However, most of previous experiments were carried out only up to $45{ }^{\circ} \mathrm{C}$. It is fair to assume that $45{ }^{\circ} \mathrm{C}$ is too low to observe the minimum CMC behavior in the CMC-temperature curve ${ }^{5}$.

In this study, polyoxyethylene sorbitan fatty acid esters (polysorbate) also known as Tween ${ }^{\circledR}$ that are non-ionic surfactants with three different fatty acid ester chains are chosen to evaluate the temperature effect on the $\mathrm{CMC}$ over a wide temperature range of $10^{\circ} \mathrm{C}$ to $80^{\circ} \mathrm{C}$. Surfactants include Polysorbate 20 or Polyoxyethylene 20 sorbitan monolaurate, Polysorbate 40 or Polyoxyethylene 20 sorbitan monopalmitate, Polysorbate 80 or Polyoxyethylene 20 sorbitan monostearate, and Polysorbate 80 or Polyoxyethylene 20 sorbitan monooleate are selected ${ }^{6}$.

Polysorbates containing 20 units of oxyethylene are hydrophilic nonionic surfactants and they are used widely as emulsifying agents in the preparation of stable oil-in-water pharmaceutical emulsions. They may also be used as solubilizing agents for a variety of substances including essential oils and oil-soluble vitamins, and as wetting agents in the formulation of oral and parenteral suspensions ${ }^{7}$.

There is intense interest in determining the thermodynamic parameters of micellization in aqueous solutions, namely, the standard Gibbs free energy of micellization, $\Delta G^{\circ}{ }_{m}$, the enthalpy of micellization, $\Delta H^{\circ}{ }_{m}$ and the entropy of micellization, $\Delta S^{\circ}{ }_{m}$. This is because they quantify the relative importance of hydrophobic interactions, surfactant-water contact and (for ionic surfactants) head-group repulsion. These parameters can be derived from the temperature dependence of the CMC, for example, by application of the Van't Hoff equation ${ }^{8}$. When CMC is measured at various temperatures, the thermodynamic potentials of micellization can also be determined. $\Delta G_{m}^{\circ}$ may be obtained from the equation ${ }_{\Delta G_{m}^{o}}^{o}=-\frac{R T \ln K}{n}$ where $K$ and $n$ being the equilibrium constant and association number, respectively. $K$ is expressed rigorously in terms of the activities and can be approximated in terms of concentrations of the constituents, one of which is the concentration $X_{C M C}$ at the CMC, in mole fraction units. The enthalpy and entropy of micelle formation are then evaluated according to the phase separation model ${ }^{9}$. 
To determine $\Delta H^{\circ}{ }_{m}$ using $\Delta G^{\circ}$ and the Gibbs-Helmholtz equation, the temperature dependence of $\Delta G^{\circ}$ m should be known so that its partial derivatives with respect to $T$ are calculated. This signifies that $\mathrm{X}_{\mathrm{CMC}}$ should be described as a function of temperature and this has been done by expressing $\mathrm{X}_{\mathrm{CMC}}$ as polynomials of temperature. However, such description of $\mathrm{X}_{\mathrm{CMC}}$ is not theory-based and therefore a theory-based and rigorous equation is desired ${ }^{10}$.

\section{Experimental}

The nonionic surfactants Tween-20, -40 and -80 were obtained from Sigma Chemical Co. (St. Louis, MO). Products were used as such without any further purification. Triply distilled and deionized water was employed in the preparation of solutions. Solutions were kept in a double-walled Pyrex vessel thermostatically controlled at a prescribed temperature. Identical glass containers and volumes were used and the container wall was kept at a minimum to reduce the effects due to adsorption on the container.

Since it was found that the surface tension decreases with time, Surface tension data were taken 24 hour, after placing the plate on the dispersion surface, to ensure the steadystate response had reached. It is believed that this dynamic behavior of surface tension is mainly due to the slow mass transfer of surfactant molecules from the bulk solution onto the interface and the slow rearrangement of surfactant molecular configuration at the interface.

\section{Evaluating surface tension}

The surface tension of solutions was determined by means of DuNouys ring platinum (diameter: $19.6 \mathrm{~mm}$, thickness: $0.1 \mathrm{~mm}$ ) on Krüss tensiometer K100. Temperature was controlled with a jacket linked to a water circulating system $\left( \pm 0.1{ }^{\circ} \mathrm{C}\right)$. The results show accuracy within $\pm 0.1 \mathrm{mNm}^{-1}$. The results $( \pm 2 \%)$ were the average of four measurements. To prevent the contamination of the solution from dust in the air during the operation, the vessel had a cover with a hole only allowing a small ring (DuNouys ring) to go through, and the whole vessel was placed inside a closed sample chamber of the surface tensiometer. The CMC values were taken from the sharp breaks in the surface tension vs. logarithms of surfactant concentration plots. The $\mathrm{CMC}$, values are expressed in $\mathrm{mol} / \mathrm{mL}$.

\section{Results and Discussion}

\section{Evaluation of the surface tension of the surfactants}

The surfactants surface tension was measured as a function of their concentration at several different temperatures, as shown in Figure 1. The CMC for three surfactants was then considered as the concentration at the sharp break.

For each surfactant, as the system temperature increases, the CMC initially decreases and then increases, as shown in Figure 2. Owing to the smaller probability of hydrogen bond formation at higher temperatures, the initial decrease of the CMC with temperature is a consequence of the decreased hydrophilicity of the surfactant molecules. In other words, the increase in temperature causes the reduction in hydration of the hydrophilic oxyethylene group, which favour micellization. Consequently, as the temperature increases the micellization onset occurs at lower concentrations. On the other hand, dissolving the surfactant molecules in water makes the hydrophobic group distorts the water structure. Additionally increase in temperature also causes an increase in the breakdown of the structured water surrounding the hydrophobic groups, which disfavours micellization. Consequently, the onset of micellization tends to occur at higher concentrations as the temperature increases. Therefore, as the temperature increases further, the effect of the hydrophobic groups begins to exert an influence and finally predominates as the 
$\mathrm{CMC}$ reaches a minimum value and finally increases with temperature. The temperatures of minimum CMC are found to be 43,42 and $40{ }^{\circ} \mathrm{C}$ for Polysorbate 20,40 and 80 respectively. Note that the temperature of minimum CMC is systematically higher as the fatty acid chain length decreases, in accord with the result of Chen et al ${ }^{5}$.

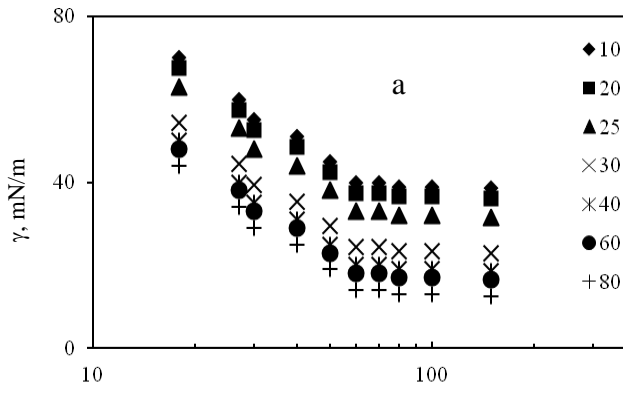

C, $10^{-6} \mathrm{~mol} / \mathrm{lit}$

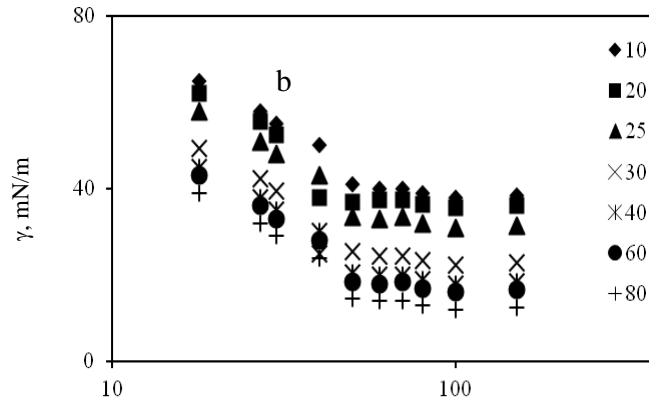

C, $10^{-6} \mathrm{~mol} / \mathrm{lit}$

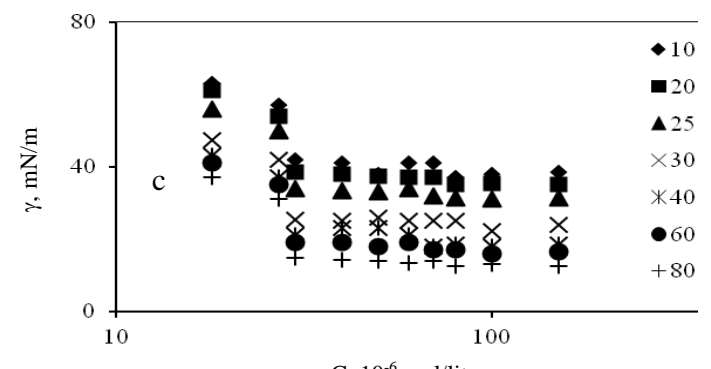

C, $10^{-6} \mathrm{~mol} / \mathrm{lit}$

Figure 1. The variation of surface tension as a function of surfactant concentration in aqueous solution (a) Polysorbate-20 (b) Polysorbate-40 and (c) Polysorbate-80 at different temperatures $\left(10-80^{\circ} \mathrm{C}\right)$.

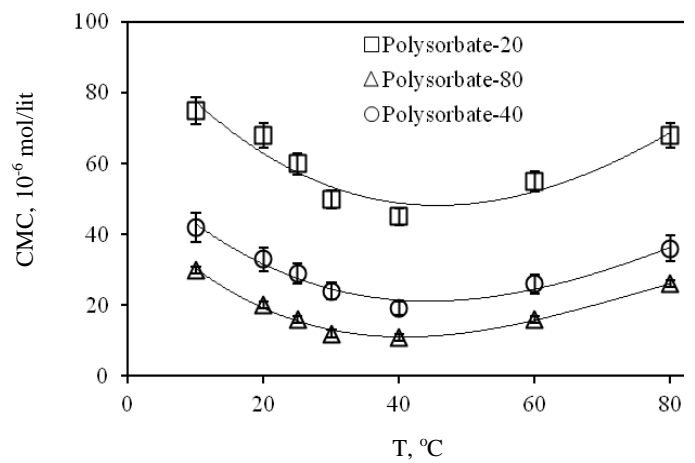

Figure 2. The variation of $\mathrm{CMC}$ as a function of temperature for different surfactants.

It is obvious that for all three surfactant solutions the surface tension $\gamma_{\mathrm{CMC}}$ decreases monotonically as the temperature increases (Figure 3) and Polysorbate-80 is the most surface active among the three systems over the whole temperature range. It should be further noted that the decrease in surface tension $\gamma_{\mathrm{CMC}}$ is more pronounced for surfactants 
with a longer fatty acid chain length, as shown in Figure 3, due to an increase of the rupture of hydrogen bonds.

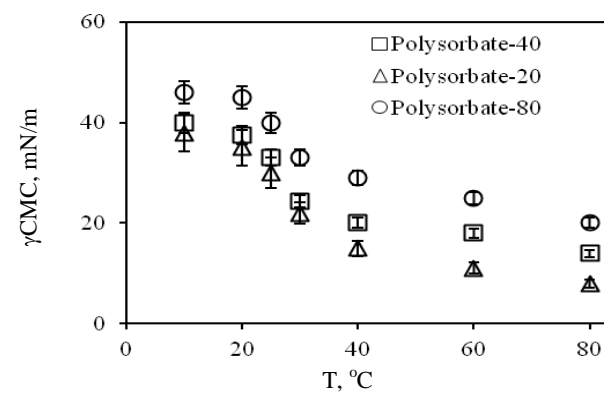

Figure 3. The variation of surface tension at $\mathrm{CMC}$ as a function of temperature for different surfactants.

It is well understood that there exists hydrogen bonding between water and surfactant molecules. While the temperature increases, some of the existing hydrogen bonds would rupture due to thermal fluctuation, which makes the surfactant molecules more hydrophobic, and thus the surface tension decreases. Therefore, the dehydration effect would be somewhat more pronounced for longer chain length surfactants since the surfactant molecule binds considerably more water molecules.

\section{Surface pressure}

Inoue et al. ${ }^{11}$ found a decrease of surface pressure $\left(\pi_{\mathrm{CMC}}\right)$, defined by $\pi_{\mathrm{CMC}}=\gamma_{0}-\gamma_{\mathrm{CMC}}$, against rising temperature for some polyoxyethylene nonionic surfactants. The symbol $\gamma_{0}$ is the surface tension of water. Figure 4 shows that the surface pressure $\pi_{\mathrm{CMC}}$ decreases monotonically as the temperature increases over a wide temperature range $\left(10-80{ }^{\circ} \mathrm{C}\right)$ for three surfactants. For the Polysorbate- 80 , the surface pressure $\pi_{\mathrm{CMC}}$ remains constant at low temperatures, and then decreases with further increase in temperature. However, for the Polysorbate-20, the surface pressure $\pi_{\mathrm{CMC}}$ remains almost constant over the temperature range, except that at high temperature a slight decrease of surface pressure $\pi_{\mathrm{CMC}}$ is observed. Our results are consistent with those of Chen et al. ${ }^{5}$ in the whole temperatures range.

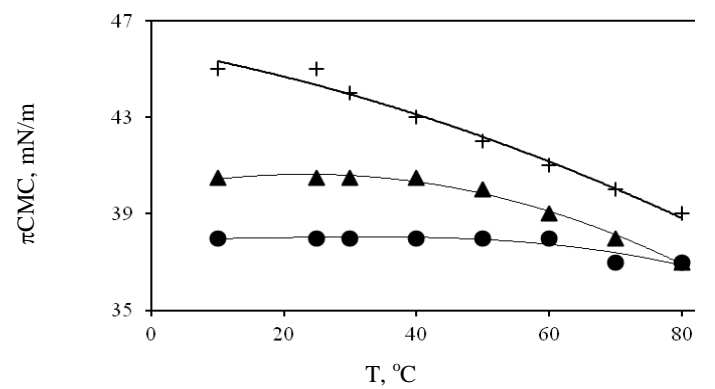

Figure 4. The variation of surface pressure $\boldsymbol{\pi}_{\mathrm{CMC}}$ as a function of temperature for the surfactants Polysorbate-80 (+), Polysorbate-40 ( $\mathbf{A})$ and Polysorbate-20 (•)

\section{Determination of thermodynamic parameters}

The CMC temperature dependence may be used to estimate the enthalpy and entropy of micellization. According to the phase separation model ${ }^{12}$ and the mass action model ${ }^{13}$, the standard Gibbs free energy of micellization per mole of a surfactant monomer is given by Eq. (1) where $\mathrm{X}_{\mathrm{CMC}}$ is the mole fraction of surfactant in aqueous solution at the CMC. 


$$
\Delta G_{m}^{o}=R T \ln x_{C M C}
$$

As the aggregation number of surfactant monomers is not too small and not strongly temperature dependent ${ }^{13}$, the enthalpy of micellization can be obtained by applying the Gibbs-Helmholtz Eq. to (1):

$$
\Delta H_{m}^{o}=-T^{2} x \frac{\partial\left(\Delta G_{m}^{o} / T\right)}{\partial T}=-R T^{2} x \frac{\partial\left(\Delta G_{m}^{o} / T\right)}{\partial T}
$$

To evaluate the enthalpy of micellization, the CMCs are first correlated by a polynomial equation where constants a, b, c, and d are determined by least-squares regression analyses:

$$
\operatorname{Ln} x_{\mathrm{CMC}}(\mathrm{T})=\mathrm{a}+\mathrm{bT}+\mathrm{cT}^{2}+\mathrm{dT}^{2}
$$

The enthalpy of micellization is calculated by substituting Eq. (3) into Eq. (2):

$$
\Delta H_{m}^{o}=-R T_{2}(b+2 c T)+3 d T^{2}
$$

As the Gibbs free energy and the enthalpy of micellization are obtained, the entropy of micelle formation can be determined by:

$$
\Delta S_{m}^{o}=\frac{1}{T\left(\Delta H_{m}^{o}-\Delta G_{m}^{o}\right)}
$$

Figure 5 shows the temperature dependence of $\Delta G^{\circ}{ }_{m}$ and $-T \Delta S^{\circ}{ }_{m}$ for polysorbate-20, polysorbate-40 and polysorbate- 80 in an aqueous system. At low temperatures, the micellization process is endothermic $\left(\Delta H_{m}^{\circ}>0\right)$ and becomes exothermic $\left(\Delta H_{m}^{\circ}<0\right)$ at high temperatures. Our results show that the negative value of Gibbs free energy of micellization is mainly due to the large positive value of entropy, especially at low temperatures. Therefore, the micellization is an entropy-driven process.

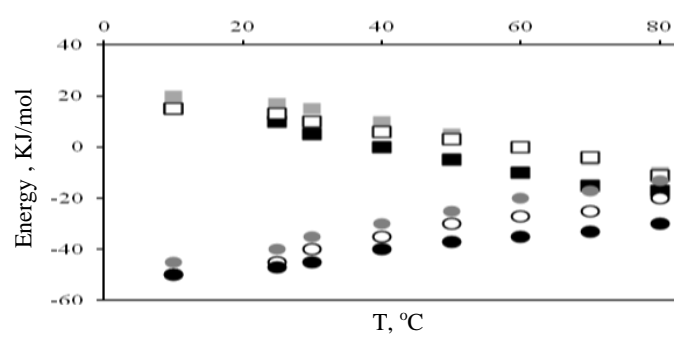

Figure 5. The variations of $\Delta H^{\circ}{ }_{m}$ (squares) and $-T \Delta S^{\circ}{ }_{m}$ (circles) as a function of temperature for three surfactants (Polysorbate-40, Polysorbate-20 and Polysorbate-80 are show by white, black, and grey respectively).

The increase in entropy of micellization in an aqueous medium can be explained from two aspects: First the iceberg formation ${ }^{5}$ of the water molecules surrounding the surfactant molecules would increase the system order, here the micellization process by removing the surfactant molecules from the aqueous medium to the micelle would certainly increase the entropy of the system simply due to the rupture of iceberg; second the degree of rotational 
freedom of the hydrophobic chain of surfactant molecules in the non-polar interior of the micelle is much larger than that in the aqueous medium ${ }^{8}$; in other words, the configurational entropy of hydrophobic chain of surfactant molecules is increased when the surfactant molecules are removed from the aqueous medium to the micelle.

It is found that $\Delta G^{\circ}{ }_{m}$ decreases monotonically as the temperature increases over the whole temperature range from $10{ }^{\circ} \mathrm{C}$ to $80{ }^{\circ} \mathrm{C}$. Both $\Delta H^{\circ}{ }_{m}$ and $\Delta S^{\circ}{ }_{m}$ appear to be decrease monotonically with an increase in temperature. According to Eq. (5), these two properties $\left(\Delta H^{\circ}{ }_{m}\right.$ and $\Delta S^{\circ}{ }_{m}$ ) have opposite effects on $\Delta G^{\circ}{ }_{m}$. Therefore, $\Delta G^{\circ}{ }_{m}$ depends on the relative amount of the changes in $\Delta H^{\circ}{ }_{m}$ and $\Delta S^{\circ}{ }_{m}$. There is a good linearity correlation between $\Delta H^{\circ}{ }_{m}$ and $\Delta S_{m}^{\circ}$, which can be interpreted by

$$
\Delta H_{m}^{o}=\Delta H_{m}^{o}+T_{c} \Delta S_{m}^{o}
$$

Where $\mathrm{T}_{\mathrm{c}}$ is the compensation temperature and $\Delta H^{\circ}$ is the intercept of the compensation line. In other words, the compensation temperature is temperature dependent and slightly larger at higher temperatures, in accord with the previous findings ${ }^{9,11}$. It is well believed that the compensation temperature is a characteristic of hydrophobic interactions between solvent and solute molecules and the intercept $\Delta H^{\circ}{ }_{m}$ is the measure of solute-solute interaction $^{5}$.

\section{Conclusion}

The compensation temperature was found to be $42{ }^{\circ} \mathrm{C}$ by linear regression over the whole temperature range and for all three surfactant systems together.

\section{Conflicting interests}

The authors declare that they have no conflicting interests.

\section{References}

1. Sinnko J, Martin's "Physical Pharmacy and Pharmaceutical Sciences," $5^{\text {th }}$ Ed., Lippincott Williams \& Wilkins, Baltimore, 2006, Chapter 9.

2. Goto A, Takemoto M and Endo F, Bull Chem Soc Jpn., 1985, 58, 247-251.

3. Rosen J M, "Surfactants and Interfacial Phenomena," $3^{\text {rd }}$ Ed., John Wiley \& Sons, New York, 2004, Chapter 3.

4. Hall D G, "Nonionic Surfactants Physical Chemistry," Marcel Dekker, New York, 1967, Chapter 13.

5. Chen L, Lin S Y and Huang C, Colloids Surf A Physicochem Eng Asp., 1998, 135, 175-181.

6. Rowe R, Sheskey P and Owen S, "Handbook of Pharmaceutical Excipients," 5th ed., Pharmaceutical Press, London, 2006, Chapter 32.

7. Jiao J, Adv Drug Deliv Rev., 2008, 60, 1663-1673.

8. Shimizu S, Pires P, Loh W and Seoud O, Colloid Polym Sci., 2004, 282, 1026-1032.

9. Kim H and Lim K, Colloids Surf A Physicochem Eng Asp., 2004, 235, 121-128.

10. Razavizadeh B M, Mousavi-Khoshdel M and Gharibi H, J Colloid Interface Sci., 2004, 276, 197-207.

11. Inoue T, Nakashima K and Suzuki M, J Oleo Sci., 51, 753-760 (2002).

12. Akbas H, Iscan M and Sidim T, J Surfactant Deterg., 2000, 3(1), 77-81.

13. Rafati A A, Gharibi H and Rezaie-Sameti M, J Mol Liq., 2004, 111, 109-116. 


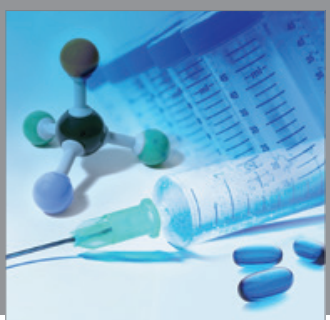

International Journal of

Medicinal Chemistry

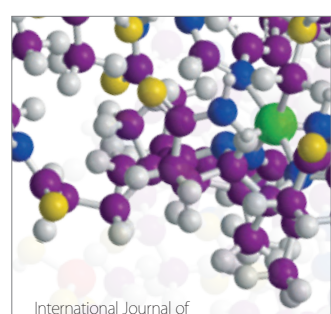

Carbohydrate Chemistry

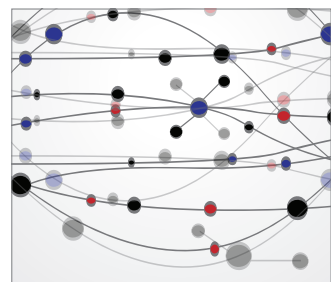

The Scientific World Journal
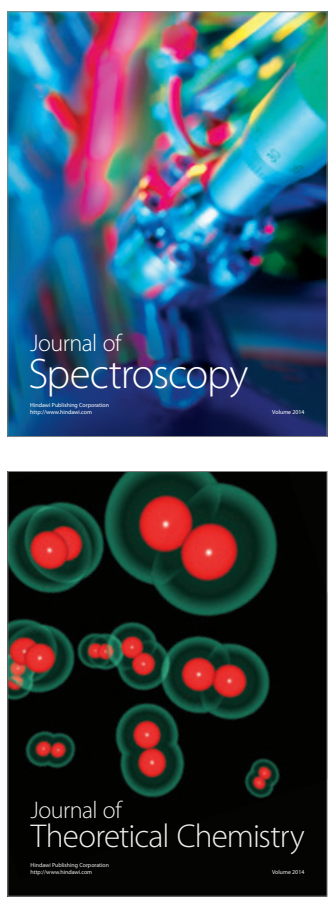
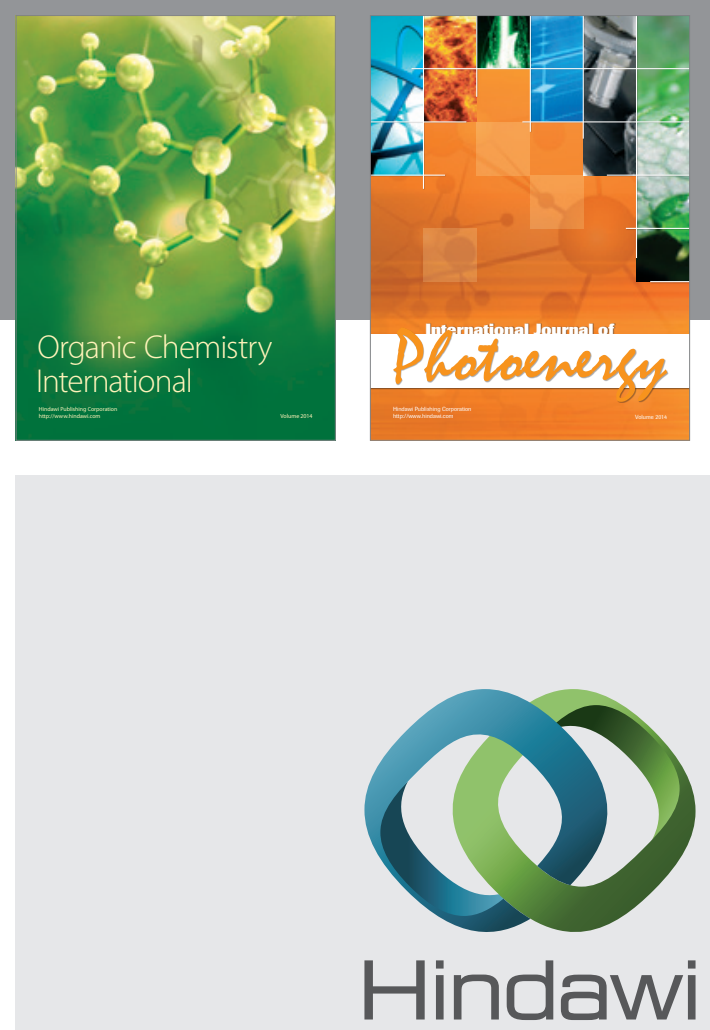

Submit your manuscripts at

http://www.hindawi.com
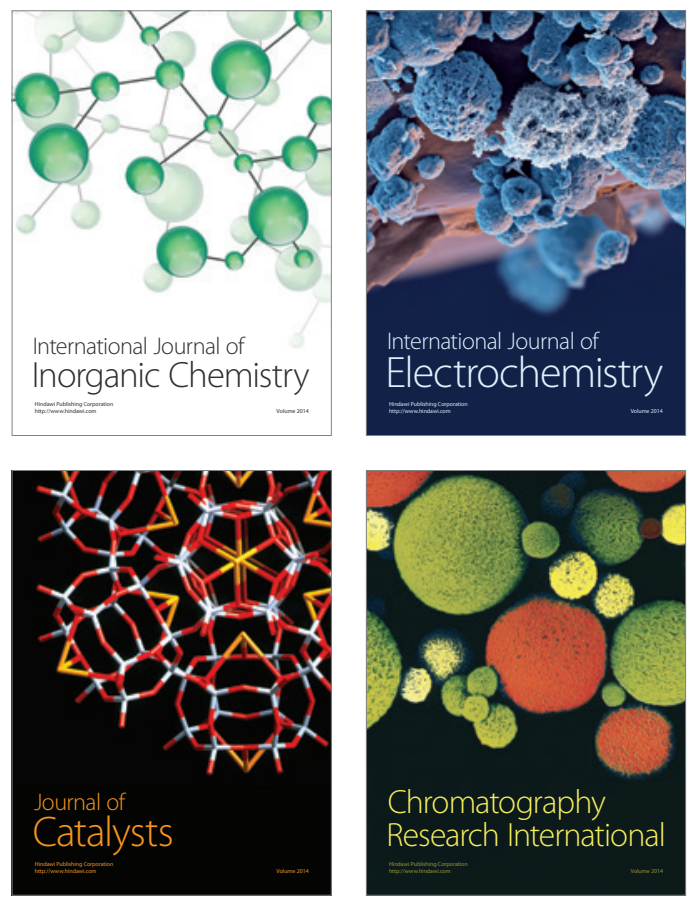
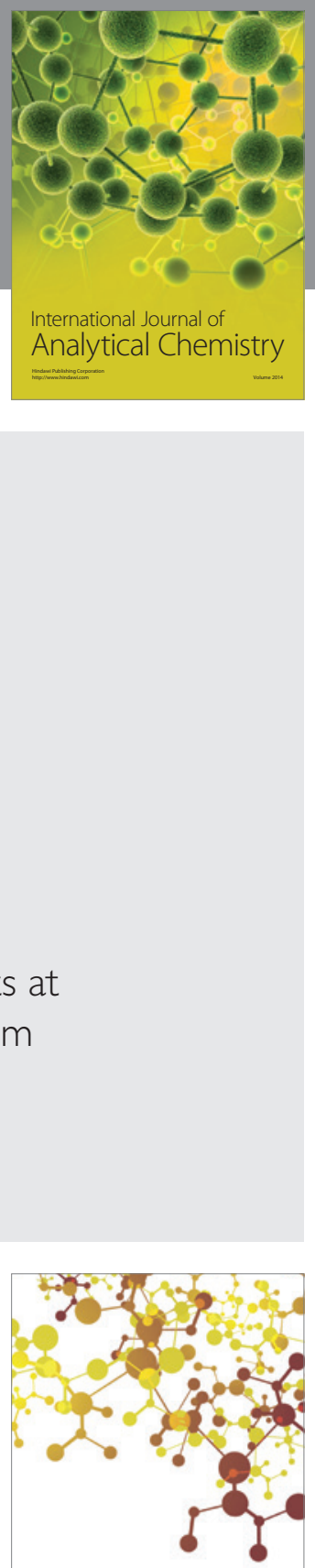

Journal of

Applied Chemistry
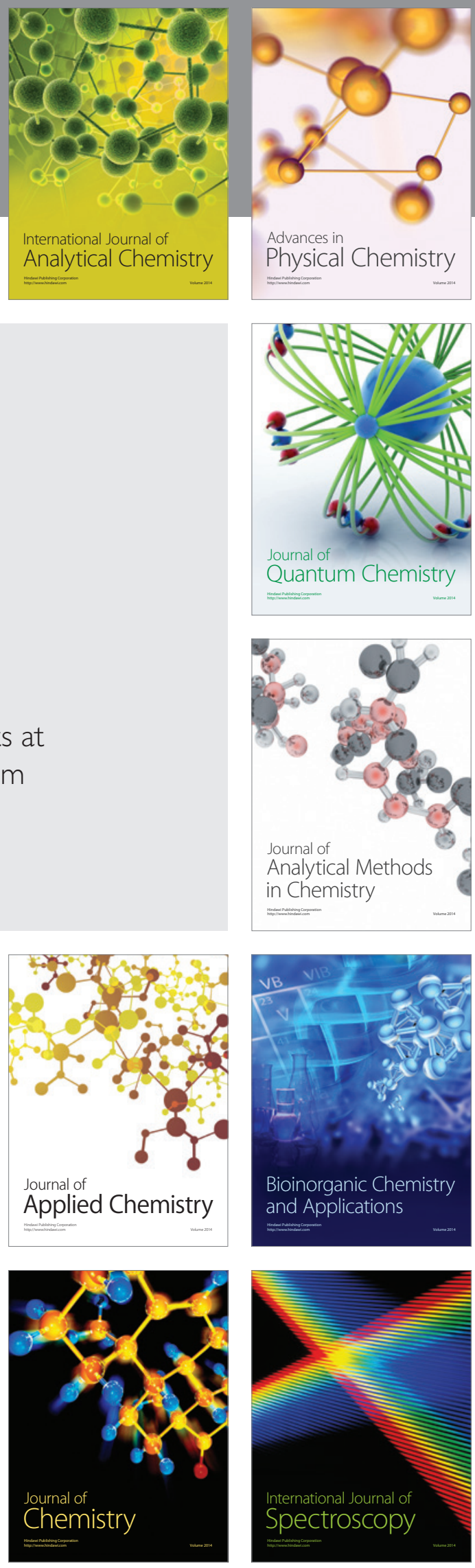